Research Article

\title{
On the Elliptic Problems Involving Multisingular Inverse Square Potentials and Concave-Convex Nonlinearities
}

\author{
Tsing-San Hsu \\ Center for General Education, Chang Gung University, Kwei-Shan, Tao-Yuan 333, Taiwan \\ Correspondence should be addressed to Tsing-San Hsu, tshsu@mail.cgu.edu.tw \\ Received 15 October 2010; Accepted 19 January 2011 \\ Academic Editor: W. A. Kirk
}

Copyright (c) 2011 Tsing-San Hsu. This is an open access article distributed under the Creative Commons Attribution License, which permits unrestricted use, distribution, and reproduction in any medium, provided the original work is properly cited.

A semilinear elliptic problem $\left(E_{\curlywedge}\right)$ with concave-convex nonlinearities and multiple Hardy-type terms is considered. By means of a variational method, we establish the existence and multiplicity of positive solutions for problem $\left(E_{\curlywedge}\right)$.

\section{Introduction and Main Results}

In this paper, we consider the following semilinear elliptic problem:

$$
\begin{aligned}
-\Delta u-\sum_{i=1}^{k} \frac{\mu_{i}}{\left|x-a_{i}\right|^{2}} u & =Q(x)|u|^{2^{*}-2} u+\lambda|u|^{q-2} u, \quad x \in \Omega, \\
u & =0, \quad x \in \partial \Omega,
\end{aligned}
$$

where $\Omega \subset \mathbb{R}^{N}(N \geq 3)$ is a smooth bounded domain such that the different points $a_{i} \in \Omega, i=$ $1,2, \ldots, k, k \geq 2,0 \leq \mu_{i}<\bar{\mu} \triangleq((N-2) / 2)^{2}, \lambda>0,1 \leq q<2,2^{*} \triangleq 2 N /(N-2)$ is the critical Sobolev exponent, and $Q(x)$ is a positive bounded function on $\bar{\Omega}$.

Problem $\left(E_{\lambda}\right)$ is related to the well-known Hardy inequality (see $\left.[1,2]\right)$ :

$$
\int_{\Omega} \frac{|u|^{2}}{|x-a|^{2}} d x \leq \frac{1}{\bar{\mu}} \int_{\Omega}|\nabla u|^{2} d x, \quad \forall u \in H_{0}^{1}(\Omega), a \in \Omega .
$$


In this paper, for $\sum_{i=1}^{k} \mu_{i} \in[0, \bar{\mu})$, we use $H \triangleq H_{0}^{1}(\Omega)$ to denote the completion of $C_{0}^{\infty}(\Omega)$ with respect to the norm

$$
\|u\|=\|u\|_{H}=\left(\int_{\Omega}\left(|\nabla u|^{2}-\sum_{i=1}^{k} \frac{\mu_{i} u^{2}}{\left|x-a_{i}\right|^{2}}\right) d x\right)^{1 / 2} .
$$

By (1.1), this norm is equivalent to the usual norm $\left(\int_{\Omega}|\nabla u|^{2} d x\right)^{1 / 2}$.

The function $u \in H$ is said to be solution of problem $\left(E_{\lambda}\right)$ if $u$ satisfies

$$
\int_{\Omega}\left(\nabla u \nabla v-\sum_{i=1}^{k} \frac{\mu_{i}}{\left|x-a_{i}\right|^{2}} u v-Q(x)|u|^{2^{*}-2} u v-\lambda|u|^{q-2} u v\right) d x=0, \quad \forall v \in H,
$$

and, by the standard elliptic regularity argument, we have that $u \in C^{2}\left(\Omega \backslash\left\{a_{1}, a_{2}, \ldots, a_{k}\right\}\right) \cap$ $C^{1}\left(\bar{\Omega} \backslash\left\{a_{1}, a_{2}, \ldots, a_{k}\right\}\right)$.

The energy functional corresponding to problem $\left(E_{\curlywedge}\right)$ is defined as follows:

$$
J_{\lambda}(u) \triangleq \frac{1}{2} \int_{\Omega}\left(|\nabla u|^{2}-\sum_{i=1}^{k} \frac{\mu_{i} u^{2}}{\left|x-a_{i}\right|^{2}}\right) d x-\frac{1}{2^{*}} \int_{\Omega}|u|^{2^{*}} d x-\frac{\lambda}{q} \int_{\Omega}|u|^{q} d x
$$

then $J_{\lambda}(u)$ is well defined on $H$ and belongs to $C^{1}(H, \mathbb{R})$. The solutions of problem $\left(E_{\curlywedge}\right)$ are then the critical points of the functional $J_{\lambda}$.

It should be mentioned that, for $0 \in \Omega, \lambda>0,1 \leq q<2,0 \leq \mu<\bar{\mu}, 0 \leq s<2$ and $2^{*}(s)=2(N-s) /(N-2)$ is the critical Sobolev-Hardy exponent. Note that $2^{*}(0)=2^{*}$, the following semilinear elliptic problem:

$$
\begin{gathered}
-\Delta u-\frac{\mu}{|x|^{2}} u=Q(x) \frac{|u|^{2^{*}(s)-2}}{|x|^{s}} u+\lambda|u|^{q-2} u, \quad x \in \Omega, \\
u=0, \quad x \in \partial \Omega,
\end{gathered}
$$

had been extensively studied, and the existence and multiplicity results of positive solutions had been obtained; see [3-7] and references therein.

For the case $k \geq 2$, our problem $\left(E_{\curlywedge}\right)$ can be regarded as a perturbation problem of the following semilinear elliptic problem:

$$
\begin{gathered}
-\Delta u-\sum_{i=1}^{k} \frac{\mu_{i}}{\left|x-a_{i}\right|^{2}} u=Q(x)|u|^{2^{*}-2} u, \quad x \in \Omega, \\
u=0, \quad x \in \partial \Omega .
\end{gathered}
$$

In [8], by using Morse iteration, the authors studied the asymptotic behavior of solutions for problem (1.6); by critical point theory, the authors also proved the existence of nontrivial solutions to problem (1.6). On the other hand, the authors in [9] also studied problem (1.6); they discussed the corresponding Rayleigh quotient and gave both sufficient and necessary 
conditions on masses and location of singularities for the minimum to be achieved. In [9], both the case of the whole $\mathbb{R}^{N}$ and bounded domains are taken into account.

To proceed, we make some motivations of the present paper. In [6], the authors studied more general problem than problem (1.5) with $\mu \in[0, \bar{\mu}), s=0$, and they proved that there exists $\Lambda>0$ such that problem (1.5) has at least two positive solutions for all $\lambda \in(0, \Lambda)$. A natural question is whether the above results remain true for problem $\left(E_{\curlywedge}\right)$ with multisingular inverse square potentials. In recent work [10], the author studied problem (1.1) with $Q(x) \equiv 1$ on $\bar{\Omega}$ and showed that there exists $\Lambda>0$ such that problem (1.1) has at least two positive solutions for all $\lambda \in(0, \Lambda)$. In this paper, we continue the study of [10] by considering the more general function $Q(x)$ instead of $Q(x) \equiv 1$ and extend the results of [10] to the more general function $Q(x)$.

For $0 \leq \mu_{i}<\bar{\mu}$ and $a_{i} \in \Omega, i=1,2, \ldots, k$, we can define the best constant

$$
S_{\mu_{i}} \triangleq \inf _{u \in H \backslash\{0\}} \frac{\int_{\Omega}\left(|\nabla u|^{2}-\mu_{i}\left(u^{2} /\left|x-a_{i}\right|^{2}\right)\right) d x}{\left(\int_{\Omega}|u|^{2^{*}} d x\right)^{2 / 2^{*}}}
$$

and from [11], we get that $S_{\mu_{i}}$ is independent of $\Omega$. For $0 \leq \mu<\bar{\mu}, 0 \leq \mu_{\mathrm{i}}<\bar{\mu}$, setting

$$
\begin{gathered}
\beta \triangleq \sqrt{\bar{\mu}-\mu}, \quad \gamma \triangleq \sqrt{\bar{\mu}}+\beta, \quad \gamma^{\prime} \triangleq \sqrt{\bar{\mu}}-\beta, \\
\beta_{i} \triangleq \sqrt{\bar{\mu}-\mu_{i}}, \quad \gamma_{i} \triangleq \sqrt{\bar{\mu}}+\beta_{i}, \quad \gamma_{i}^{\prime} \triangleq \sqrt{\bar{\mu}}-\beta_{i}
\end{gathered}
$$

the authors in $[1,2]$ proved that $S_{\mu_{i}}$ is attained in $\mathbb{R}^{N}$ by the function

$$
U_{\mu_{i}}\left(x-a_{i}\right)=\frac{\left(22^{*} \beta_{i}^{2}\right)^{1 /\left(2^{*}-2\right)}}{\left|x-a_{i}\right|^{\gamma_{i}^{\prime}}\left(1+\left|x-a_{i}\right|^{\left(2^{*}-2\right) \beta_{i}}\right)^{2 /\left(2^{*}-2\right)}}
$$

and, moreover, for all $\varepsilon>0, V_{\mu_{i}, \varepsilon}^{a_{i}}(x) \triangleq \varepsilon^{(2-N) / 2} U_{\mu_{i}}\left(\left(x-a_{i}\right) / \varepsilon\right)$ solve the problem

$$
-\Delta u-\frac{\mu_{i}}{\left|x-a_{i}\right|^{2}} u=|u|^{2^{*}-2} u \text { in } \mathbb{R}^{N} \backslash\left\{a_{i}\right\}
$$

and satisfy

$$
\int_{\mathbb{R}^{N}}\left(\left|\nabla V_{\mu_{i}, \varepsilon}^{a_{i}}\right|^{2}-\mu_{i} \frac{\left|V_{\mu_{i}, \varepsilon}^{a_{i}}\right|^{2}}{\left|x-a_{i}\right|^{2}}\right) d x=\int_{\mathbb{R}^{N}}\left|V_{\mu_{i}, \varepsilon}^{a_{i}}\right|^{2^{*}} d x=S_{\mu_{i}}^{N / 2}
$$


Note that $S_{\mu}$ is a decreasing function of $\mu$ for $\mu \in[0, \bar{\mu})$ and

$$
U_{\mu_{i}}^{a_{i}}(x)=\frac{1}{\left(\left|x-a_{i}\right|^{\gamma_{k} / \sqrt{\bar{\mu}}}+\left|x-a_{i}\right|^{\gamma_{k}^{\prime} / \sqrt{\bar{\mu}}}\right)^{\sqrt{\bar{\mu}}}}
$$

also attains $S_{\mu_{i}}$ for $i=1,2, \ldots, k$.

Now we recall the following standard definition.

Assume that $X$ is a Banach space and $X^{-1}$ is the dual space of $X$. The functional $I \in C^{1}(X, \mathbb{R})$ is said to satisfy the Palais-Smale condition at level $c\left((\mathrm{PS})_{c}\right.$ in short), if every sequence $\left\{u_{n}\right\} \subset X$ satisfying $I\left(u_{n}\right) \rightarrow c$ and $I^{\prime}\left(u_{n}\right) \rightarrow 0$ in $X^{-1}$ has a convergent subsequence.

In this paper, we will take $I=J_{\lambda}$ and $X=H$. To proceed, we need the following assumptions:

$\left(\mathscr{\ell}_{1}\right)$ there exists an $l \in\{1,2, \ldots, k\}$ such that

$$
S_{\mu_{l}}^{N / 2} Q\left(a_{l}\right)^{(2-N) / 2}=\min \left\{S_{\mu_{i}}^{N / 2} Q\left(a_{i}\right)^{(2-N) / 2}, i=1,2, \ldots, k\right\},
$$

$\left(\mathscr{\ell}_{2}\right) Q(x)$ is a positive bounded function on $\bar{\Omega}$, and there exists an $x_{0} \in \Omega$ such that $Q\left(x_{0}\right)$ is a strict local maximum. Furthermore, there exists $\tau>\left(\sqrt{\bar{\mu}-\mu_{l}} N\right) / \sqrt{\bar{\mu}}$ such that

$$
\begin{gathered}
Q\left(x_{0}\right)=Q_{M}=\max _{\bar{\Omega}} Q(x), \\
Q(x)-Q\left(x_{0}\right)=o\left(\left|x-x_{0}\right|^{\tau}\right) \quad \text { as } x \longrightarrow x_{0}, \\
Q(x)-Q\left(a_{l}\right)=o\left(\left|x-a_{l}\right|^{\tau}\right) \quad \text { as } x \longrightarrow a_{l},
\end{gathered}
$$

$\left(\mathscr{\ell}_{3}\right) 0 \leq \mu_{i}<\bar{\mu}$ for every $i=1,2, \ldots, k$ and $\sum_{i=1}^{k} \mu_{i}<\bar{\mu}$.

We define the following constants:

$$
\begin{gathered}
S \triangleq \inf _{u \in H_{0}^{1}(\Omega) \backslash\{0\}} \frac{\int_{\Omega}\left(|\nabla u|^{2}-\sum_{i=1}^{k} \mu_{i}\left(u^{2} /\left|x-a_{i}\right|^{2}\right)\right) d x}{\left(\int_{\Omega}|u|^{2^{*}} d x\right)^{2 / 2^{*}}} \\
\Lambda_{0} \triangleq\left(\frac{2-q}{\left(2^{*}-q\right) Q_{M}}\right)^{(2-q) /\left(2^{*}-2\right)}\left(\frac{2^{*}-2}{2^{*}-q}\right)|\Omega|^{-\left(\left(2^{*}-q\right) / 2^{*}\right)} S^{\left(2^{*}(2-q)\right) /\left(2\left(2^{*}-2\right)\right)+q / 2}
\end{gathered}
$$

The main result of this paper is the following theorem. 
Theorem 1.1. Assume that conditions $\left(\mathscr{L}_{1}\right)-\left(\mathscr{H}_{3}\right)$ hold; then one has the following.

(i) If $\lambda \in\left(0, \Lambda_{0}\right)$, then problem $\left(E_{\curlywedge}\right)$ has at least one positive solution.

(ii) If $\lambda \in\left(0,(q / 2) \Lambda_{0}\right)$, then problem $\left(E_{\lambda}\right)$ has at least two positive solutions.

This paper is organized as follows. In Section 2, we give some properties of Nehari manifold. In Sections 3 and 4, we complete proofs of Theorem 1.1. At the end of this section, we explain some notations employed in this paper. $L^{p}\left(\Omega,\left|x-a_{i}\right|^{t}\right)$ denotes the usual weighted $L^{p}(\Omega)$ space with the weight $\left|x-a_{i}\right|^{t} .|\Omega|$ is the Lebesgue measure of $\Omega . B_{r}(x)$ is a ball centered at $x$ with radius $r$. $O\left(\varepsilon^{t}\right)$ denotes $\left|O\left(\varepsilon^{t}\right)\right| / \varepsilon^{t} \leq C$, and $o_{n}(1)$ denotes $o_{n}(1) \rightarrow 0$ as $n \rightarrow \infty$. C, $C_{i}$ will denote various positive constants and omit $d x$ in the integration for convenience.

\section{Nehari Manifold}

In this section, we will give some properties of Nehari manifold. As the energy functional $J_{\lambda}$ is not bounded below on $H$, it is useful to consider the functional on the Nehari manifold

$$
\mathcal{M}_{\lambda}=\left\{u \in H \backslash\{0\}:\left\langle J_{\lambda}^{\prime}(u), u\right\rangle=0\right\} .
$$

Thus, $u \in \mathcal{M}_{\curlywedge}$ if and only if

$$
\left\langle J_{\lambda}^{\prime}(u), u\right\rangle=\|u\|^{2}-\int_{\Omega} Q(x)|u|^{2^{*}}-\lambda \int_{\Omega}|u|^{q}=0 .
$$

Note that $\mathcal{M}_{\lambda}$ contains every nonzero solution of problem $\left(E_{\curlywedge}\right)$. Moreover, we have the following results.

Lemma 2.1. The energy functional $J_{\lambda}$ is coercive and bounded below on $\boldsymbol{M}_{\lambda}$.

Proof. If $u \in \mathcal{M}_{\lambda}$, then by (1.15), (2.2), and Hölder inequality,

$$
\begin{aligned}
J_{\lambda}(u) & =\frac{2^{*}-2}{22^{*}}\|u\|^{2}-\lambda\left(\frac{2^{*}-q}{2^{*} q}\right) \int_{\Omega}|u|^{q} \\
& \geq \frac{1}{N}\|u\|^{2}-\lambda\left(\frac{2^{*}-q}{2^{*} q}\right)|\Omega|^{\left(2^{*}-q\right) / 2^{*}} S^{-q / 2}\|u\|^{q} .
\end{aligned}
$$

Thus, $J_{\lambda}$ is coercive and bounded below on $\mathcal{M}_{\lambda}$.

The Nehari manifold is closely linked to the behavior of the function of the form $\varphi_{u}: t \rightarrow J_{\lambda}(t u)$ for $t>0$. Such maps are known as fibering maps and were introduced by 
Drábek and Pohozaev in [12] and are also discussed by Brown and Zhang [13]. If $u \in H$, we have

$$
\begin{aligned}
& \varphi_{u}(t)=\frac{t^{2}}{2}\|u\|^{2}-\frac{t^{2^{*}}}{2^{*}} \int_{\Omega} Q(x)|u|^{2^{*}}-\lambda \frac{t^{q}}{q} \int_{\Omega}|u|^{q}, \\
& \varphi_{u}^{\prime}(t)=t\|u\|^{2}-t^{2^{*}-1} \int_{\Omega} Q(x)|u|^{2^{*}}-\lambda t^{q-1} \int_{\Omega}|u|^{q}, \\
& \varphi_{u}^{\prime \prime}(t)=\|u\|^{2}-\left(2^{*}-1\right) t^{2^{*}-2} \int_{\Omega} Q(x)|u|^{2^{*}}-\lambda(q-1) t^{q-2} \int_{\Omega}|u|^{q} .
\end{aligned}
$$

It is easy to see that

$$
t \varphi_{u}^{\prime}(t)=\|t u\|^{2}-\int_{\Omega} Q(x)|t u|^{2^{*}}-\lambda \int_{\Omega}|t u|^{q},
$$

and so, for $u \in H \backslash\{0\}$ and $t>0, \varphi_{u}^{\prime}(t)=0$ if and only if $t u \in \mathcal{M}_{\lambda}$, that is, the critical points of $\varphi_{u}$ correspond to the points on the Nehari manifold. In particular, $\varphi_{u}^{\prime}(1)=0$ if and only if $u \in \mathcal{M}_{\lambda}$. Thus, it is natural to split $\mathcal{M}_{\lambda}$ into three parts corresponding to local minima, local maxima, and points of inflection. Accordingly, we define

$$
\begin{aligned}
\mathcal{M}_{\lambda}^{+} & =\left\{u \in \mathcal{M}_{\curlywedge}: \varphi_{u}^{\prime \prime}(1)>0\right\}, \\
\mathcal{M}_{\lambda}^{0} & =\left\{u \in \mathcal{M}_{\curlywedge}: \varphi_{u}^{\prime \prime}(1)=0\right\}, \\
\mathcal{M}_{\lambda}^{-} & =\left\{u \in \mathcal{M}_{\curlywedge}: \varphi_{u}^{\prime \prime}(1)<0\right\}
\end{aligned}
$$

and note that, if $u \in \mathcal{M}_{\curlywedge}$, that is, $\varphi_{u}^{\prime}(1)=0$, then

$$
\begin{aligned}
\varphi_{u}^{\prime \prime}(1) & =(2-q)\|u\|^{2}-\left(2^{*}-q\right) \int_{\Omega} Q(x)|u|^{2^{*}} \\
& =\lambda\left(2-2^{*}\right)\|u\|^{2}-\left(q-2^{*}\right) \int_{\Omega}|u|^{q}
\end{aligned}
$$

We now derive some basic properties of $\mathcal{M}_{\lambda}^{+}, \mathcal{M}_{\lambda}^{0}$, and $\mathcal{M}_{\lambda}^{-}$.

Lemma 2.2. Assume that $u_{0}$ is a local minimizer for $J_{\lambda}$ on $\boldsymbol{M}_{\lambda}$ and $u_{0} \notin \mathcal{M}_{\lambda}^{0}$. Then $J_{\lambda}^{\prime}\left(u_{0}\right)=0$ in $H^{-1}$.

Proof. Our proof is almost the same as that in Brown-Zhang [13, Theorem 2.3] (or see Binding et al. [14]).

Moreover, we have the following result.

Lemma 2.3. If $\lambda \in\left(0, \Lambda_{0}\right)$, then $\mathcal{M}_{\lambda}^{0}=\emptyset$, where $\Lambda_{0}$ is the same as in (1.16). 
Proof. Suppose the contrary. Then there exists $\lambda \in\left(0, \Lambda_{0}\right)$ such that $\mathcal{M}_{\lambda}^{0} \neq \emptyset$. Then, for $u \in \mathcal{M}_{\lambda}^{0}$ by (1.15) and (2.7), we have that

$$
\frac{2-q}{2^{*}-q}\|u\|^{2}=\int_{\Omega} Q(x)|u|^{2^{*}} \leq Q_{M} S^{-2^{*} / 2}\|u\|^{2^{*}}
$$

and so

$$
\|u\| \geq\left(\frac{2-q}{\left(2^{*}-q\right) Q_{M}}\right)^{1 /\left(2^{*}-2\right)} S^{2^{*} /\left(2\left(2^{*}-2\right)\right)}
$$

Similarly, using (1.15), (2.8), and Hölder inequality, we have that

$$
\|u\|^{2}=\lambda \frac{2^{*}-q}{2^{*}-2} \int_{\Omega}|u|^{q} \leq \lambda \frac{2^{*}-q}{2^{*}-2}|\Omega|^{\left(2^{*}-q\right) / 2^{*}} S^{-q / 2}\|u\|^{q},
$$

which implies that

$$
\|u\| \leq\left(\lambda \frac{2^{*}-q}{2^{*}-2}|\Omega|^{\left(2^{*}-q\right) / 2^{*}}\right)^{1 /(2-q)} S^{-q /(2(2-q))} .
$$

Hence, we must have

$$
\lambda \geq\left(\frac{2-q}{\left(2^{*}-q\right) Q_{M}}\right)^{(2-q) /\left(2^{*}-2\right)}\left(\frac{2^{*}-2}{2^{*}-q}\right)|\Omega|^{-\left(2^{*}-q\right) / 2^{*}} S^{\left(2^{*}(2-q)\right) /\left(2\left(2^{*}-2\right)\right)+(q / 2)}=\Lambda_{0}
$$

which is a contradiction. This completes the proof.

In order to get a better understanding of the Nehari manifold and fibering maps, we consider the function $\psi_{u}: \mathbb{R}^{+} \rightarrow \mathbb{R}$ defined by

$$
\psi_{u}(t)=t^{2-q}\|u\|^{2}-t^{2^{*}-q} \int_{\Omega} Q(x)|u|^{2^{*}} \quad \text { for } t>0
$$

Clearly $t u \in \mathcal{M}_{\lambda}$ if and only if $\psi_{u}(t)=\lambda \int_{\Omega}|u|^{q}$. Moreover,

$$
\psi_{u}^{\prime}(t)=(2-q) t^{1-q}\|u\|^{2}-\left(2^{*}-q\right) t^{2^{*}-q-1} \int_{\Omega} Q(x)|u|^{2^{*}} \text { for } t>0
$$

and so it is easy to see that, if $t u \in \mathcal{M}_{\lambda}$, then $t^{q-1} \psi_{u}^{\prime}(t)=\varphi_{u}^{\prime \prime}(t)$. Hence, $t u \in \mathcal{M}_{\lambda}^{+}$(or $t u \in \mathcal{M}_{\lambda}^{-}$) if and only if $\psi_{u}^{\prime}(t)>0\left(\right.$ or $\left.\psi_{u}^{\prime}(t)<0\right)$. 
For $u \in H \backslash\{0\}$, by (2.15), $\psi_{u}$ has a unique critical point at $t=t_{\max }(u)$, where

$$
t_{\max }(u)=\left(\frac{(2-q)\|u\|^{2}}{\left(2^{*}-q\right) \int_{\Omega} Q(x)|u|^{2^{*}}}\right)^{1 /\left(2^{*}-2\right)}>0
$$

and clearly $\psi_{u}$ is strictly increasing on $\left(0, t_{\max }(u)\right)$ and strictly decreasing on $\left(t_{\max }(u), \infty\right)$ with $\lim _{t \rightarrow \infty} \psi_{u}(t)=-\infty$. Moreover, if $\lambda \in\left(0, \Lambda_{0}\right)$, then

$$
\begin{aligned}
\psi_{u}\left(t_{\max }(u)\right) & =\left[\left(\frac{2-q}{2^{*}-q}\right)^{(2-q) /\left(2^{*}-2\right)}-\left(\frac{2-q}{2^{*}-q}\right)^{\left(2^{*}-q\right) /\left(2^{*}-2\right)}\right] \frac{\|u\|^{\left(2\left(2^{*}-q\right)\right) /\left(2^{*}-2\right)}}{\left(\int_{\Omega} Q(x)|u|^{2^{*}}\right)^{(2-q) /\left(2^{*}-2\right)}} \\
& =\|u\|^{q}\left(\frac{2^{*}-2}{2^{*}-q}\right)\left(\frac{2-q}{2^{*}-q}\right)^{(2-q) /\left(2^{*}-2\right)}\left(\frac{\|u\|^{2^{*}}}{\int_{\Omega} Q(x)|u|^{2^{*}}}\right)^{(2-q) /\left(2^{*}-2\right)} \\
& \geq\|u\|^{q}\left(\frac{2^{*}-2}{2^{*}-q}\right)\left(\frac{2-q}{\left(2^{*}-q\right) Q_{M}}\right)^{(2-q) /\left(2^{*}-2\right)} S^{\left(2^{*}(2-q)\right) /\left(2\left(2^{*}-2\right)\right)} \\
& >\lambda|\Omega|^{\left(2^{*}-q\right) / 2^{*}} S^{-q / 2}\|u\|^{q} \\
& \geq \lambda \int_{\Omega}|u|^{q} .
\end{aligned}
$$

Therefore, we have the following lemma.

Lemma 2.4. Let $\lambda \in\left(0, \Lambda_{0}\right)$. For each $u \in H \backslash\{0\}$, one has the following:

(i) there exist unique $0<t^{+}=t^{+}(u)<t_{\max }(u)<t^{-}=t^{-}(u)$ such that $t^{+} u \in \mathcal{M}_{\lambda^{+}}, t^{-} u \in$ $\mathcal{M}_{\lambda^{-}}^{-} \varphi_{u}$ is decreasing on $\left(0, t^{+}\right)$, increasing on $\left(t^{+}, t^{-}\right)$and decreasing on $\left(t^{-}, \infty\right)$

$$
J_{\lambda}\left(t^{+} u\right)=\inf _{0 \leq t \leq t_{\max }(u)} J_{\lambda}(t u), \quad J_{\lambda}\left(t^{-} u\right)=\sup _{t \geq t^{+}} J_{\lambda}(t u),
$$

(ii) $\mathcal{M}_{\lambda}^{-}=\left\{u \in H \backslash\{0\}:(1 /\|u\|) t^{-}(u /\|u\|)=1\right\}$,

(iii) there exists a continuous bijection between $U=\{u \in H \backslash\{0\}:\|u\|=1\}$ and $\mathcal{M}_{\lambda}^{-}$. In particular, $t^{-}$is a continuous function for $u \in H \backslash\{0\}$.

Proof. For the proof see $\mathrm{Wu}[15$, Lemma 2.6].

\section{Existence of Ground State}

First, we remark that it follows from Lemma 2.3 that

$$
\mathcal{M}_{\lambda}=\mathcal{M}_{\lambda}^{+} \cup \mathcal{M}_{\lambda}^{-}
$$


for all $\lambda \in\left(0, \Lambda_{0}\right)$. Furthermore, by Lemma 2.4 it follows that $\mathcal{M}_{\lambda}^{+}$and $\mathcal{M}_{\lambda}^{-}$are nonempty, and by Lemma 2.1 we may define

$$
\alpha_{\lambda}=\inf _{u \in \mathcal{M}_{\lambda}} J_{\lambda}(u), \quad \alpha_{\lambda}^{+}=\inf _{u \in \mathscr{M}_{\lambda}^{+}} J_{\lambda}(u), \quad \alpha_{\lambda}^{-}=\inf _{u \in \mathscr{M}_{\lambda}^{-}} J_{\lambda}(u)
$$

Then we get the following result.

Theorem 3.1. One has the following.

(i) If $\lambda \in\left(0, \Lambda_{0}\right)$, then one has $\alpha_{\lambda}^{+}<0$.

(ii) If $\lambda \in\left(0,(q / 2) \Lambda_{0}\right)$, then $\alpha_{\lambda}^{-}>d_{0}$ for some $d_{0}>0$.

In particular, for each $\lambda \in\left(0,(q / 2) \Lambda_{0}\right)$, one has $\alpha_{\lambda}^{+}=\alpha_{\lambda}$.

Proof. (i) Let $u \in \mathcal{M}_{\lambda}^{+}$. By (2.7),

$$
\frac{2-q}{2^{*}-q}\|u\|^{2}>\int_{\Omega} Q(x)|u|^{2^{*}}
$$

and so

$$
\begin{aligned}
J_{\lambda}(u) & =\left(\frac{1}{2}-\frac{1}{q}\right)\|u\|^{2}+\left(\frac{1}{q}-\frac{1}{2^{*}}\right) \int_{\Omega} Q(x)|u|^{2^{*}} \\
& <\left[\left(\frac{1}{2}-\frac{1}{q}\right)+\left(\frac{1}{q}-\frac{1}{2^{*}}\right)\left(\frac{2-q}{2^{*}-q}\right)\right]\|u\|^{2} \\
& =-\frac{\left(2^{*}-2\right)(2-q)}{22^{*} q}\|u\|^{2}<0 .
\end{aligned}
$$

Therefore, $\alpha_{\lambda}^{+}<0$.

(ii) Let $u \in \mathcal{M}_{\lambda}^{-}$. By (2.7),

$$
\frac{2-q}{2^{*}-q}\|u\|^{2}<\int_{\Omega} Q(x)|u|^{2^{*}} .
$$

Moreover, by (1.15), we have that

$$
\int_{\Omega} Q(x)|u|^{2^{*}} \leq Q_{M} S^{-2^{*} / 2}\|u\|^{2^{*}}
$$

This implies that

$$
\|u\|>\left(\frac{2-q}{\left(2^{*}-q\right) Q_{M}}\right)^{1 /\left(2^{*}-2\right)} S^{N / 4} \quad \forall u \in \mathcal{M}_{\lambda}^{-}
$$


By (2.3) and (3.7), we have that

$$
\begin{aligned}
J_{\lambda}(u) \geq & \|u\|^{q}\left[\frac{1}{N}\|u\|^{2-q}-\lambda\left(\frac{2^{*}-q}{2^{*} q}\right) S^{-q / 2}|\Omega|^{\left(2^{*}-q\right) / 2^{*}}\right] \\
> & \left(\frac{2-q}{\left(2^{*}-q\right) Q_{M}}\right)^{q /\left(2^{*}-2\right)} \\
& \times S^{N q / 4}\left[\frac{1}{N}\left(\frac{2-q}{\left(2^{*}-q\right) Q_{M}}\right)^{(2-q) /\left(2^{*}-2\right)} S^{((2-q) N) / 4}-\lambda\left(\frac{2^{*}-q}{2^{*} q}\right) S^{-q / 2}|\Omega|^{\left(2^{*}-q\right) / 2^{*}}\right] .
\end{aligned}
$$

Thus, if $\lambda \in\left(0,(q / 2) \Lambda_{0}\right)$, then

$$
J_{\lambda}(u)>d_{0} \quad \forall u \in \mathcal{M}_{\lambda^{\prime}}^{-}
$$

for some positive constant $d_{0}$. This completes the proof.

Remark 3.2. (i) If $\lambda \in\left(0, \Lambda_{0}\right)$, then by (1.15), (2.8), and Hölder inequality, for each $u \in \mathcal{M}_{\lambda}^{+}$, we have that

$$
\begin{aligned}
\|u\|^{2} & <\lambda \frac{2^{*}-q}{2^{*}-2} \int_{\Omega}|u|^{q} \\
& \leq \lambda \frac{2^{*}-q}{2^{*}-2} S^{-q / 2}|\Omega|^{\left(2^{*}-q\right) / 2^{*}}\|u\|^{q} \\
& \leq \Lambda_{0} \frac{2^{*}-q}{2^{*}-2} S^{-q / 2}|\Omega|^{\left(2^{*}-q\right) / 2^{*}}\|u\|^{q},
\end{aligned}
$$

and so

$$
\|u\|<\left(\Lambda_{0} \frac{2^{*}-q}{2^{*}-2} S^{-q / 2}|\Omega|^{\left(2^{*}-q\right) / 2^{*}}\right)^{1 /(2-q)} \quad \forall u \in \mathcal{M}_{\lambda}^{+}
$$

(ii) If $\lambda \in\left(0,(q / 2) \Lambda_{0}\right)$, then by Lemma 2.4 (i) and Theorem 3.1 (ii), for each $u \in \mathcal{M}_{\mathcal{\lambda}}^{-}$ we have that

$$
J_{\lambda}(u)=\sup _{t \geq 0} J_{\lambda}(t u)
$$

Now, we use the Ekeland variational principle [16] to get the following results.

Proposition 3.3. (i) If $\lambda \in\left(0, \Lambda_{0}\right)$, then there exists a (PS) $\alpha_{\lambda}$ sequence $\left\{u_{n}\right\} \subset \mathcal{M}_{\lambda}$ in $H$ for $J_{\lambda}$. (ii) If $\lambda \in\left(0,(q / 2) \Lambda_{0}\right)$, then there exists a (PS) $\alpha_{\alpha_{\lambda}^{-}}$sequence $\left\{u_{n}\right\} \subset \mathcal{M}_{\lambda}^{-}$in $H$ for $J_{\lambda}$. Proof. The proof is almost the same as that in $\mathrm{Wu}[17$, Proposition 9]. 
Now, we establish the existence of a local minimum for $J_{\lambda}$ on $\mathcal{M}_{\lambda}^{+}$.

Theorem 3.4. Assume that condition ( $\mathscr{\ell})$ holds. If $\lambda \in\left(0, \Lambda_{0}\right)$, then $J_{\lambda}$ has a minimizer $u_{\lambda}$ in $\mathcal{M}_{\lambda}^{+}$ and it satisfies the following:

(i) $J_{\lambda}\left(u_{\curlywedge}\right)=\alpha_{\lambda}=\alpha_{\lambda}^{+}$

(ii) $u_{\curlywedge}$ is a positive solution of problem $\left(E_{\curlywedge}\right)$,

(iii) $\left\|u_{\lambda}\right\| \rightarrow 0$ as $\lambda \rightarrow 0^{+}$.

Proof. By Proposition 3.3 (i), there is a minimizing sequence $\left\{u_{n}\right\}$ for $J_{\lambda}$ on $\mathcal{M}_{\lambda}$ such that

$$
J_{\lambda}\left(u_{n}\right)=\alpha_{\lambda}+o_{n}(1), \quad J^{\prime}\left(u_{n}\right)=o_{n}(1) \quad \text { in } H^{-1}(\Omega) .
$$

Since $J_{\lambda}$ is coercive on $\boldsymbol{M}_{\lambda}$ (see Lemma 2.1), we get that $\left\{u_{n}\right\}$ is bounded in $H$. Going if necessary to a subsequence, we can assume that there exists $u_{\lambda} \in H$ such that

$$
\begin{aligned}
& u_{n} \rightarrow u_{\curlywedge} \text { weakly in } H, \\
& u_{n} \longrightarrow u_{\curlywedge} \text { almost everywhere in } \Omega, \\
& u_{n} \longrightarrow u_{\curlywedge} \text { strongly in } L^{s}(\Omega) \forall 1 \leq s<2^{*} .
\end{aligned}
$$

Thus, we have that

$$
\lambda \int_{\Omega}\left|u_{n}\right|^{q}=\lambda \int_{\Omega}\left|u_{\lambda}\right|^{q}+o_{n}(1) \quad \text { as } n \longrightarrow \infty
$$

First, we claim that $u_{\curlywedge}$ is a nonzero solution of problem $\left(E_{\curlywedge}\right)$. By (3.13) and (3.14), it is easy to see that $u_{\lambda}$ is a solution of problem $\left(E_{\lambda}\right)$. From $u_{n} \in \mathcal{M}_{\lambda}$ and (2.2), we deduce that

$$
\lambda \int_{\Omega}\left|u_{n}\right|^{q}=\frac{q\left(2^{*}-2\right)}{2\left(2^{*}-q\right)}\left\|u_{n}\right\|^{2}-\frac{2^{*} q}{2^{*}-q} J_{\lambda}\left(u_{n}\right)
$$

Let $n \rightarrow \infty$ in (3.16); by (3.13), (3.15), and $\alpha_{\lambda}<0$, we get

$$
\lambda \int_{\Omega}\left|u_{\lambda}\right|^{q} \geq-\frac{2^{*} q}{2^{*}-q} \alpha_{\curlywedge}>0
$$

Thus, $u_{\lambda} \in \mathcal{M}_{\lambda}$ is a nonzero solution of problem $\left(E_{\lambda}\right)$. Now we prove that $u_{n} \rightarrow u_{\curlywedge}$ strongly in $H$ and $J_{\lambda}\left(u_{\lambda}\right)=\alpha_{\lambda}$. By (3.16), if $u \in \mathcal{M}_{\lambda}$, then

$$
J_{\lambda}(u)=\frac{1}{N}\|u\|^{2}-\lambda \frac{2^{*}-q}{2^{*} q} \int_{\Omega}|u|^{q} .
$$


In order to prove that $J_{\lambda}\left(u_{\lambda}\right)=\alpha_{\lambda}$, it suffices to recall that $u_{n}, u_{\lambda} \in \mathcal{M}_{\lambda}$, by (3.18) and applying Fatou's lemma to get

$$
\begin{aligned}
\alpha_{\lambda} & \leq J_{\lambda}\left(u_{\lambda}\right)=\frac{1}{N}\left\|u_{\lambda}\right\|^{2}-\lambda \frac{2^{*}-q}{2^{*} q} \int_{\Omega}\left|u_{\lambda}\right|^{q} \\
& \leq \liminf _{n \rightarrow \infty}\left(\frac{1}{N}\left\|u_{n}\right\|^{2}-\lambda \frac{2^{*}-q}{2^{*} q} \int_{\Omega}\left|u_{n}\right|^{q}\right) \\
& \leq \liminf _{n \rightarrow \infty} J_{\lambda}\left(u_{n}\right)=\alpha_{\lambda} .
\end{aligned}
$$

This implies that $J_{\lambda}\left(u_{\lambda}\right)=\alpha_{\curlywedge}$ and $\lim _{n \rightarrow \infty}\left\|u_{n}\right\|^{2}=\left\|u_{\lambda}\right\|^{2}$. Let $v_{n}=u_{n}-u_{\lambda}$; then Brézis-Lieb's lemma [18] implies that

$$
\left\|v_{n}\right\|^{2}=\left\|u_{n}\right\|^{2}-\left\|u_{\curlywedge}\right\|^{2}+o_{n}(1)
$$

Therefore, $u_{n} \rightarrow u_{\lambda}$ strongly in $H$. Moreover, we have $u_{\lambda} \in \mathcal{M}_{\lambda}^{+}$. On the contrary, if $u_{\lambda} \in \mathcal{M}_{\lambda}^{-}$, then, by Lemma 2.4, there are unique $t_{0}^{+}$and $t_{0}^{-}$such that $t_{0}^{+} u_{\lambda} \in \mathcal{M}_{\lambda}^{+}$and $t_{0}^{-} u_{\lambda} \in \mathcal{M}_{\lambda}^{-}$. In particular, we have $t_{0}^{+}<t_{0}^{-}=1$. Since

$$
\frac{d}{d t} J_{\lambda}\left(t_{0}^{+} u_{\curlywedge}\right)=0, \quad \frac{d^{2}}{d t^{2}} J_{\lambda}\left(t_{0}^{+} u_{\curlywedge}\right)>0
$$

there exists $t_{0}^{+}<\bar{t} \leq t_{0}^{-}$such that $J_{\lambda}\left(t_{0}^{+} u_{\lambda}\right)<J_{\lambda}\left(\bar{t} u_{\curlywedge}\right)$. By Lemma 2.4 (i),

$$
J_{\lambda}\left(t_{0}^{+} u_{\lambda}\right)<J_{\lambda}\left(\bar{t} u_{\curlywedge}\right) \leq J_{\lambda}\left(t_{0}^{-} u_{\curlywedge}\right)=J_{\lambda}\left(u_{\curlywedge}\right)
$$

which is a contradiction. Since $J_{\lambda}\left(u_{\lambda}\right)=J_{\lambda}\left(\left|u_{\lambda}\right|\right)$ and $\left|u_{\lambda}\right| \in \mathcal{M}_{\lambda}^{+}$, by Lemma 2.2, we may assume that $u_{\curlywedge}$ is a nonzero nonnegative solution of problem $\left(E_{\curlywedge}\right)$. By Harnack inequality [19], we deduce that $u_{\lambda}>0$ in $\Omega$. Finally, by (3.10), we have that

$$
\left\|u_{\lambda}\right\|^{2-q}<\lambda \frac{2^{*}-q}{2^{*}-2}|\Omega|^{\left(2^{*}-q\right) / 2^{*}} S^{-q / 2}
$$

and so $\left\|u_{\curlywedge}\right\| \rightarrow 0$ as $\lambda \rightarrow 0^{+}$.

\section{Proof of Theorem 1.1}

In this section, we will establish the existence of the second positive solution of problem $\left(E_{\curlywedge}\right)$ by proving that $J_{\lambda}$ attains a local minimum on $\mathcal{M}_{\lambda}^{-}$.

Lemma 4.1. If $\left\{u_{n}\right\} \subset H$ is a (PS) sequence for $J_{\lambda}$, then $\left\{u_{n}\right\}$ is bounded in $H$.

Proof. The argument is similar to that of [10, Lemma 4.1], and here we omit the details. 
We recall that

$$
S_{\mu_{i}} \triangleq \inf _{u \in H \backslash\{0\}} \frac{\int_{\Omega}\left(|\nabla u|^{2}-\mu_{i}\left(u^{2} /\left|x-a_{i}\right|^{2}\right)\right) d x}{\left(\int_{\Omega}|u|^{2^{*}} d x\right)^{2 / 2^{*}}} .
$$

Lemma 4.2. Assume that conditions $\left(\mathscr{H}_{1}\right)-\left(\mathscr{L}_{3}\right)$ holds. If $\left\{u_{n}\right\} \subset H$ is a (PS) $)_{c}$ sequence for $J_{\lambda}$ with

$$
0<c<c^{*} \triangleq \frac{1}{N} \min \left\{\frac{S_{\mu_{l}}^{N / 2}}{Q\left(a_{l}\right)^{(N-2) / 2}}, \frac{S_{0}^{N / 2}}{Q_{M}^{(N-2) / 2}}\right\},
$$

then there exists a subsequence of $\left\{u_{n}\right\}$ converging weakly to a nonzero solution of problem $\left(E_{\curlywedge}\right)$.

Proof. Let $\left\{u_{n}\right\} \subset H$ be a (PS) $)_{c}$ sequence for $J_{\lambda}$ with $c \in\left(0, c^{*}\right)$. We know from Lemma 4.1 that $\left\{u_{n}\right\}$ is bounded in $H$, and then there exists a subsequence of $\left\{u_{n}\right\}$ (still denoted by $\left.\left\{u_{n}\right\}\right)$ and $u_{0} \in H$ such that

$$
\begin{aligned}
& u_{n} \rightarrow u_{0} \text { weakly in } H, \\
& u_{n} \rightarrow u_{0} \text { weakly in } L^{2}\left(\Omega,\left|x-a_{i}\right|^{-2}\right) \text { for } 1 \leq i \leq k, \\
& u_{n} \rightarrow u_{0} \text { weakly in } L^{2^{*}}(\Omega), \\
& u_{n} \longrightarrow u_{0} \text { almost everywhere in } \Omega, \\
& u_{n} \longrightarrow u_{0} \text { strongly in } L^{S}(\Omega) \forall 1 \leq s<2^{*} .
\end{aligned}
$$

It is easy to see that $J_{\lambda}^{\prime}\left(u_{0}\right)=0$ and

$$
\lambda \int_{\Omega}\left|u_{n}\right|^{q}=\lambda \int_{\Omega}\left|u_{0}\right|^{q}+o_{n}(1)
$$

Next we verify that $u_{0} \not \equiv 0$. Arguing by contradiction, we assume that $u_{0} \equiv 0$. By the concentration compactness principle (see $[20,21]$ ), there exist a subsequence, still denoted by $\left\{u_{n}\right\}$, at most countable set 2 , a set of different points $\left\{x_{j}\right\}_{j \in 2} \subset \Omega \backslash\left\{a_{1}, a_{2}, \ldots, a_{k}\right\}$, nonnegative real numbers $\widetilde{\mu_{x_{j}}}, \widetilde{v_{x_{j}}}, j \in \mathcal{2}$, and nonnegative real numbers $\widetilde{\mu_{a_{i}}}, \widetilde{r_{a_{i}}}, \widetilde{v_{a_{i}}},(1 \leq$ $i \leq k)$ such that

$$
\begin{aligned}
& \left|\nabla u_{n}\right|^{2} \rightarrow d \tilde{\mu} \geq\left|\nabla u_{0}\right|^{2}+\sum_{j \in \mathcal{\partial}} \widetilde{\mu_{x_{j}}} \delta_{x_{j}}+\sum_{i=1}^{k} \widetilde{\mu_{a_{i}}} \delta_{a_{i}}, \\
& \frac{u_{n}^{2}}{\left|x-a_{i}\right|^{2}} \rightarrow d \tilde{\gamma}=\frac{u_{0}^{2}}{\left|x-a_{i}\right|^{2}}+\widetilde{\gamma_{a_{i}}} \delta_{a_{i}}, \\
& \left|u_{n}\right|^{2^{*}} \rightarrow d \tilde{v}=\left|u_{0}\right|^{2^{*}}+\sum_{j \in \mathcal{\partial}} \widetilde{v_{x_{j}}} \delta_{x_{j}}+\sum_{i=1}^{k} \widetilde{v_{a_{i}}} \delta_{a_{i},}
\end{aligned}
$$


where $\delta_{x}$ is the Dirac mass at $x$. By the Sobolev-Hardy inequalities, we infer that

$$
S_{\mu_{i}}{\widetilde{v_{a_{i}}}}^{2 / 2^{*}} \leq \widetilde{\mu_{a_{i}}}-\mu_{i} \widetilde{\gamma_{a_{i}}}, \quad 1 \leq i \leq k
$$

We claim that 2 is finite and, for any $j \in \mathcal{\partial}$, either

$$
\widetilde{v_{x_{j}}}=0 \quad \text { or } \quad Q\left(x_{j}\right) \widetilde{v_{x_{j}}} \geq \frac{S_{0}^{N / 2}}{Q_{M}^{(N-2) / N}}
$$

In fact, let $\varepsilon>0$ be small enough such that $a_{i} \notin B_{\varepsilon}\left(x_{j}\right)$ for all $1 \leq i \leq k$ and $B_{\varepsilon}\left(x_{i}\right) \cap$ $B_{\varepsilon}\left(x_{j}\right)=\varnothing$ for $i \neq j, i, j \in 2$. Let $\phi_{\varepsilon}^{j}$ be a smooth cut-off function centered at $x_{j}$ such that $0 \leq \phi_{\varepsilon}^{j} \leq 1, \phi_{\varepsilon}^{j}=1$ for $\left|x-x_{j}\right| \leq \varepsilon / 2, \phi_{\varepsilon}^{j}=0$ for $\left|x-x_{j}\right| \geq \varepsilon$ and $\left|\nabla \phi_{\varepsilon}^{j}\right| \leq 4 / \varepsilon$. Then

$$
\begin{gathered}
\lim _{\varepsilon \rightarrow 0} \lim _{n \rightarrow \infty} \int_{\Omega}\left|\nabla u_{n}\right|^{2} \phi_{\varepsilon}^{j}=\lim _{\varepsilon \rightarrow 0} \int_{\Omega} \phi_{\varepsilon}^{j} d \tilde{\mu} \geq \lim _{\varepsilon \rightarrow 0}\left(\int_{\Omega}\left|\nabla u_{0}\right|^{2} \phi_{\varepsilon}^{j}+\widetilde{\mu_{x_{j}}}\right)=\widetilde{\mu_{x_{j}}}, \\
\lim _{\varepsilon \rightarrow 0} \lim _{n \rightarrow \infty} \int_{\Omega} \frac{u_{n}^{2}}{\left|x-a_{i}\right|^{2}} \phi_{\varepsilon}^{j}=\lim _{\varepsilon \rightarrow 0} \int_{\Omega} \phi_{\varepsilon}^{j} d \tilde{\gamma}=\lim _{\varepsilon \rightarrow 0} \int_{\Omega} \frac{u_{0}^{2}}{\left|x-a_{i}\right|^{2}} \phi_{\varepsilon}^{j}=0, \\
\lim _{\varepsilon \rightarrow 0} \lim _{n \rightarrow \infty} \int_{\Omega} Q(x)\left|u_{n}\right|^{2^{*}} \phi_{\varepsilon}^{j}=\lim _{\varepsilon \rightarrow 0} \int_{\Omega} Q(x) \phi_{\varepsilon}^{j} d \tilde{v}=\lim _{\varepsilon \rightarrow 0}\left(\int_{\Omega} Q(x)\left|u_{0}\right|^{2^{*}} \phi_{\varepsilon}^{j}+Q\left(x_{j}\right) \widetilde{v_{x_{j}}}\right)=Q\left(x_{j}\right) \widetilde{v_{x_{j}}}, \\
\lim _{\varepsilon \rightarrow 0} \lim _{n \rightarrow \infty} \int_{\Omega} u_{n} \nabla u_{n} \nabla \phi_{\varepsilon}^{j}=0 .
\end{gathered}
$$

Thus we have that

$$
0=\lim _{\varepsilon \rightarrow 0} \lim _{n \rightarrow \infty}\left\langle J_{\lambda}^{\prime}\left(u_{n}\right), u_{n} \phi_{\varepsilon}^{j}\right\rangle \geq \widetilde{\mu_{x_{j}}}-Q\left(x_{j}\right) \widetilde{{v_{j}}_{j}}
$$

By the Sobolev inequality, $S_{0}{\widetilde{v_{x_{j}}}}^{2 / 2^{*}} \leq \widetilde{\mu_{x_{j}}}$ for $j \in 2$; hence we deduce that

$$
\widetilde{v_{x_{j}}}=0 \quad \text { or } \quad Q\left(x_{j}\right) \widetilde{v_{x_{j}}} \geq \frac{S_{0}^{N / 2}}{Q_{M}^{(N-2) / 2}}
$$

which implies that 2 is finite.

Now we consider the possibility of concentraction at points $a_{i}(1 \leq i \leq k)$. For $\varepsilon>0$ be small enough such that $x_{j} \notin B_{\varepsilon}\left(a_{i}\right)$ for all $j \in \partial$ and $B_{\varepsilon}\left(a_{i}\right) \cap B_{\varepsilon}\left(a_{j}\right)=\varnothing$ for $i \neq j$ and 
$1 \leq i, j \leq k$. Let $\varphi_{\varepsilon}^{i}$ be a smooth cut-off function centered at $a_{i}$ such that $0 \leq \varphi_{\varepsilon}^{i} \leq 1, \varphi_{\varepsilon}^{i}=1$ for $\left|x-a_{i}\right| \leq \varepsilon / 2, \varphi_{\varepsilon}^{i}=0$ for $\left|x-a_{i}\right| \geq \varepsilon$ and $\left|\nabla \varphi_{\varepsilon}^{i}\right| \leq 4 / \varepsilon$. Then

$$
\begin{gathered}
\lim _{\varepsilon \rightarrow 0} \lim _{n \rightarrow \infty} \int_{\Omega}\left|\nabla u_{n}\right|^{2} \varphi_{\varepsilon}^{i}=\lim _{\varepsilon \rightarrow 0} \int_{\Omega} \varphi_{\varepsilon}^{i} d \tilde{\mu} \geq \lim _{\varepsilon \rightarrow 0}\left(\int_{\Omega}\left|\nabla u_{0}\right|^{2} \varphi_{\varepsilon}^{i}+\widetilde{\mu_{a_{i}}}\right)=\widetilde{\mu_{a_{i}}}, \\
\lim _{\varepsilon \rightarrow 0} \lim _{n \rightarrow \infty} \int_{\Omega} \frac{u_{n}^{2}}{\left|x-a_{i}\right|^{2}} \varphi_{\varepsilon}^{i}=\lim _{\varepsilon \rightarrow 0} \int_{\Omega} \varphi_{\varepsilon}^{\mathrm{i}} d \tilde{\gamma}=\lim _{\varepsilon \rightarrow 0}\left(\int_{\Omega} \frac{u_{0}^{2}}{\left|x-a_{i}\right|^{2}} \varphi_{\varepsilon}^{i}+\widetilde{r_{a_{i}}}\right)=\widetilde{r_{a_{i}}}, \\
\lim _{\varepsilon \rightarrow 0} \lim _{n \rightarrow \infty} \int_{\Omega} Q(x)\left|u_{n}\right|^{2^{*}} \varphi_{\varepsilon}^{i}=\lim _{\varepsilon \rightarrow 0} \int_{\Omega} Q(x) \varphi_{\varepsilon}^{i} d \tilde{v}=\lim _{\varepsilon \rightarrow 0}\left(\int_{\Omega} Q(x)\left|u_{0}\right|^{2^{*}} \varphi_{\varepsilon}^{i}+Q\left(a_{i}\right) \widetilde{v_{a_{i}}}\right)=Q\left(a_{i}\right) \widetilde{v_{a_{i}}}, \\
\lim _{\varepsilon \rightarrow 0} \lim _{n \rightarrow \infty} \int_{\Omega} \frac{u_{n}^{2}}{\left|x-a_{j}\right|^{2}} \varphi_{\varepsilon}^{i}=0 \quad \text { for } j \neq i, \\
\lim _{\varepsilon \rightarrow 0} \lim _{n \rightarrow \infty} \int_{\Omega} u_{n} \nabla u_{n} \nabla \varphi_{\varepsilon}^{i}=0 .
\end{gathered}
$$

Thus we have that

$$
0=\lim _{\varepsilon \rightarrow 0} \lim _{n \rightarrow \infty}\left\langle J_{\lambda}^{\prime}\left(u_{n}\right), u_{n} \varphi_{\varepsilon}^{i}\right\rangle \geq \widetilde{\mu_{a_{i}}}-\mu_{i} \widetilde{\gamma_{a_{i}}}-Q\left(a_{i}\right) \widetilde{v_{a_{i}}}
$$

From (4.6) and (4.12) we derive that

$$
S_{\mu_{i}}{\widetilde{v_{i}}}^{2 / 2^{*}} \leq Q\left(a_{i}\right) \widetilde{v_{a_{i}}}
$$

and then either $\widetilde{v_{a_{i}}}=0$ or $\widetilde{v_{a_{i}}} \geq\left(S_{\mu_{i}} / Q\left(a_{i}\right)\right)^{N / 2}$ for all $1 \leq i \leq k$.

On the other hand, from the above arguments and (4.4), we conclude that

$$
\begin{aligned}
c & =\lim _{n \rightarrow \infty}\left(J_{\lambda}\left(u_{n}\right)-\frac{1}{2}\left\langle J_{\lambda}^{\prime}\left(u_{n}\right), u_{n}\right\rangle\right) \\
& =\frac{1}{N} \lim _{n \rightarrow \infty} \int_{\Omega} Q(x)\left|u_{n}\right|^{2^{*}}+\lambda\left(\frac{1}{2}-\frac{1}{q}\right) \int_{\Omega}\left|u_{0}\right|^{q} \\
& =\frac{1}{N}\left(\int_{\Omega} Q(x)\left|u_{0}\right|^{2^{*}}+\sum_{j \in 2} Q\left(x_{j}\right) \widetilde{v_{x_{j}}}+\sum_{i=1}^{k} Q\left(a_{i}\right) \widetilde{v_{a_{i}}}\right)+\lambda\left(\frac{1}{2}-\frac{1}{q}\right) \int_{\Omega}\left|u_{0}\right|^{q} \\
& =\frac{1}{N}\left(\sum_{j \in 2} Q\left(x_{j}\right) \widetilde{v_{x_{j}}}+\sum_{i=1}^{k} Q\left(a_{i}\right) \widetilde{v_{a_{i}}}\right)+J_{\lambda}\left(u_{0}\right) .
\end{aligned}
$$


If $\widetilde{v_{a_{i}}}=\widetilde{v_{x_{j}}}=0$ for all $i \in\{1,2, \ldots, k\}$ and $j \in 2$, then $c=0$ which contradicts the assumption that $c>0$. On the other hand, if there exists an $i \in\{1,2, \ldots, k\}$ such that $\widetilde{v_{a_{i}}} \neq 0$ or there exists a $j \in \partial$ with $\widetilde{v_{x_{j}}} \neq 0$, then we infer that

$$
\begin{aligned}
c & \geq \frac{1}{N} \min \left\{\frac{S_{\mu_{1}}^{N / 2}}{Q\left(a_{1}\right)^{(N-2) / 2}}, \frac{S_{\mu_{2}}^{N / 2}}{Q\left(a_{2}\right)^{(N-2) / 2}}, \ldots, \frac{S_{\mu_{k}}^{N / 2}}{Q\left(a_{k}\right)^{(N-2) / 2}}, \frac{S_{0}^{N / 2}}{Q_{M}^{(N-2) / 2}}\right\} \\
& =\frac{1}{N} \min \left\{\frac{S_{\mu_{l}}^{N / 2}}{Q\left(a_{l}\right)^{(N-2) / 2}}, \frac{S_{0}^{N / 2}}{Q_{M}^{(N-2) / 2}}\right\} \\
& =c^{*},
\end{aligned}
$$

which also contradicts the assumption that $c<c^{*}$. Therefore $u_{0}$ is a nonzero solution of problem $\left(E_{\curlywedge}\right)$.

Lemma 4.3. Assume that conditions $\left(\mathfrak{L}_{1}\right)-\left(\mathfrak{L}_{3}\right)$ hold. Then for any $\lambda>0$, there exist $v_{\lambda} \in H_{0}^{1}(\Omega)$ such that

$$
\sup _{t \geq 0} J_{\lambda}\left(t v_{\lambda}\right)<c^{*}
$$

In particular, $\alpha_{\lambda}^{-}<c^{*}$ for all $\lambda \in\left(0, \Lambda_{0}\right)$ where $\Lambda_{0}$ is the same as in (1.16).

Proof. From $\left(\mathscr{\ell}_{2}\right)$, we know that there exist $\rho_{0}>0, \tau>\left(\sqrt{\bar{\mu}-\mu_{l}} N\right) / \sqrt{\bar{\mu}}$ such that $B_{2 \rho_{0}}\left(a_{l}\right) \subset$ $\Omega, B_{2 \rho_{0}}\left(x_{0}\right) \subset \Omega$,

$$
\begin{aligned}
& Q(x)=Q\left(a_{l}\right)+o\left(\left|x-a_{l}\right|^{\tau}\right) \quad \forall x \in B_{2 \rho_{0}}\left(a_{l}\right), \\
& Q(x)=Q_{M}+o\left(\left|x-x_{0}\right|^{\tau}\right) \quad \forall x \in B_{2 \rho_{0}}\left(x_{0}\right) .
\end{aligned}
$$

To prove this lemma, we need to distinguish the following two cases:

$$
\text { case I: } \frac{S_{\mu l}^{N / 2}}{Q\left(a_{l}\right)^{(N-2) / 2}}<\frac{S_{0}^{N / 2}}{Q_{M}^{(N-2) / 2}}, \quad \text { case II: } \frac{S_{\mu_{l}}^{N / 2}}{Q\left(a_{l}\right)^{(N-2) / 2}} \geq \frac{S_{0}^{N / 2}}{Q_{M}^{(N-2) / 2}} \text {. }
$$

We first study Case I. The definition of $c^{*}$ implies that

$$
c^{*}=\frac{S_{\mu_{l}}^{N / 2}}{N Q\left(a_{l}\right)^{(N-2) / 2}} .
$$

Motivated by some ideas of selecting cut-off functions in [22], we take such cut-off function $\eta^{a_{l}}(x)$ that satisfies $\eta^{a_{l}}(x) \in C_{0}^{\infty}\left(B_{2 \delta_{0}}\left(a_{l}\right)\right), \eta^{a_{l}}(x)=1$ for $\left|x-a_{l}\right|<\delta_{0}, \eta^{a_{l}}(x)=0$ for $\left|x-a_{l}\right|>$ 
$2 \delta_{0}, 0 \leq \eta^{a_{l}} \leq 1$ and $\left|\nabla \eta^{a_{l}}\right| \leq C$ where $0<\delta_{0}<\min \left\{(1 / 2)\left|a_{i}-a_{j}\right|, i, j=1,2, \ldots, k, i \neq j\right\}, \delta_{0} \leq$ $\rho_{0}$, and $B_{2 \delta_{0}}\left(a_{l}\right) \subset \Omega$. For $\varepsilon>0$, let

$$
u_{\mu_{l}, \varepsilon}^{a_{l}}(x)=\frac{\varepsilon^{(N-2) / 4} \eta^{a_{l}}(x)}{\left[\varepsilon\left|x-a_{l}\right|^{r_{l}^{\prime} / \sqrt{\bar{\mu}}}+\left|x-a_{l}\right|^{\gamma / \sqrt{\bar{\mu}}}\right]^{\sqrt{\bar{\mu}}}},
$$

where $\bar{\mu}=((N-2) / 2)^{2}, \gamma_{l}^{\prime}=\sqrt{\bar{\mu}}-\sqrt{\bar{\mu}-\mu_{l}}$, and $\gamma_{l}=\sqrt{\bar{\mu}}+\sqrt{\bar{\mu}-\mu_{l}}$.

We define the following functions on the interval $[0,+\infty)$ :

$$
\begin{aligned}
g(t) \triangleq & J_{\lambda}\left(t u_{\mu_{l}, \varepsilon}^{a_{l}}\right) \\
= & \frac{t^{2}}{2} \int_{\Omega}\left(\left|\nabla u_{\mu_{l}, \varepsilon}^{a_{l}}\right|^{2}-\mu_{l} \frac{\left(u_{\mu_{l}, \varepsilon}^{a_{l}}\right)^{2}}{\left|x-a_{l}\right|^{2}}\right)-\frac{t^{2^{*}}}{2^{*}} \int_{\Omega} Q(x)\left|u_{\mu_{l}, \varepsilon}^{a_{l}}\right|^{2^{*}} \\
& -\frac{t^{2}}{2} \sum_{i \neq l, i=1}^{k} \mu_{i} \int_{\Omega} \frac{\left(u_{\mu_{l}, \varepsilon}^{a_{l}}\right)^{2}}{\left|x-a_{i}\right|^{2}}-\lambda \frac{t^{q}}{q} \int_{\Omega}\left|u_{\mu_{l}, \varepsilon}^{a_{l}}\right|^{q} \\
\leq & \frac{t^{2}}{2} \int_{\Omega}\left(\left|\nabla u_{\mu_{l}, \varepsilon}^{a_{l}}\right|^{2}-\mu_{l} \frac{\left(u_{\mu_{l}, \varepsilon}^{a_{l}}\right)^{2}}{\left|x-a_{l}\right|^{2}}\right)-\frac{t^{2^{*}}}{2^{*}} \int_{\Omega} Q(x)\left|u_{\mu_{l}, \varepsilon}^{a_{l}}\right|^{2^{*}}-\lambda \frac{t^{q}}{q} \int_{\Omega}\left|u_{\mu_{l}, \varepsilon}^{a_{l}}\right|^{q}, \\
\bar{g}(t) \triangleq & \frac{t^{2}}{2} \int_{\Omega}\left(\left|\nabla u_{\mu_{l}, \varepsilon}^{a_{l}}\right|^{2}-\mu_{l} \frac{\left(u_{\mu_{l}, \varepsilon}^{a_{l}}\right)^{2}}{\left|x-a_{l}\right|^{2}}\right)-\frac{t^{2^{*}}}{2^{*}} \int_{\Omega} Q(x)\left|u_{\mu_{l}, \varepsilon}^{a_{l}}\right|^{2^{*}} .
\end{aligned}
$$

From Hsu and Lin [6, Lemma 5.3] and after a detailed calculation, we have the following estimates:

$$
\begin{aligned}
\left(\int_{\Omega} Q(x)\left|u_{\mu_{l}, \varepsilon}^{a_{l}}\right|^{2^{*}}\right)^{2 / 2^{*}} & =\left(\int_{\mathbb{R}^{N}} Q\left(a_{l}\right)\left|U_{\mu_{l}}^{a_{l}}\right|^{2^{*}}\right)^{2 / 2^{*}}+O\left(\varepsilon^{N / 2}\right), \\
\int_{\Omega}\left(\left|\nabla u_{\mu_{l}, \varepsilon}^{a_{l}}\right|^{2}-\mu_{l} \frac{\left(u_{\mu_{l}, \varepsilon}^{a_{l}}\right)^{2}}{\left|x-a_{l}\right|^{2}}\right) & =\int_{\mathbb{R}^{N}}\left(\left|\nabla U_{\mu_{l}}^{a_{l}}\right|^{2}-\mu_{l} \frac{\left(U_{\mu_{l}}^{a_{l}}\right)^{2}}{\left|x-a_{l}\right|^{2}}\right)+O\left(\varepsilon^{(N-2) / 2}\right), \\
\sup _{t \geq 0} \bar{g}(t) & =\frac{S_{\mu_{l}}^{N / 2}}{N Q\left(a_{l}\right)^{(N-2) / 2}}+O\left(\varepsilon^{(N-2) / 2}\right),
\end{aligned}
$$

where $U_{\mu_{l}}^{a_{l}}$ is defined as in (1.12).

Using the definitions of $g(t), u_{\mu_{l}, \varepsilon}^{a_{l}}$, we get

$$
g(t) \leq \frac{t^{2}}{2} \int_{\Omega}\left(\left|\nabla u_{\mu_{l}, \varepsilon}^{a_{l}}\right|^{2}-\mu_{l} \frac{\left(u_{\mu_{l}, \varepsilon}^{a_{l}}\right)^{2}}{\left|x-a_{l}\right|^{2}}\right), \quad \forall t \geq 0, \forall \lambda>0 .
$$


Combining this with (4.22), let $\varepsilon \in(0,1)$; then there exists $t_{0} \in(0,1)$ independent of $\varepsilon$ such that

$$
\sup _{0 \leq t \leq t_{0}} g(t)<\frac{S_{\mu_{l}}^{N / 2}}{N Q\left(a_{l}\right)^{(N-2) / 2}}, \quad \forall \lambda>0, \forall \varepsilon \in(0,1)
$$

Using the definitions of $g(t)$ and $u_{\mu_{l}, \varepsilon}^{a_{l}}$ and by (4.23), we have that

$$
\begin{aligned}
\sup _{t \geq t_{0}} g(t) & =\sup _{t \geq t_{0}}\left(\bar{g}(t)-\frac{t^{q}}{q} \lambda \int_{\Omega}\left|u_{\mu_{l}, \varepsilon}^{a_{l}}\right|^{q}\right) \\
& \leq \frac{S_{\mu_{l}}^{N / 2}}{N Q\left(a_{l}\right)^{(N-2) / 2}}+O\left(\varepsilon^{(N-2) / 2}\right)-\lambda \frac{t_{0}^{q}}{q} \int_{B_{\delta_{0}}\left(a_{l}\right)}\left|u_{\mu_{l}, \varepsilon}^{a_{l}}\right|^{q} .
\end{aligned}
$$

Let $0<\varepsilon \leq \delta_{0}^{\left(\gamma_{l}-\gamma_{l}^{\prime}\right) / \sqrt{\bar{\mu}}}$; then we have that

$$
\begin{aligned}
\int_{B_{\delta_{0}}\left(a_{l}\right)}\left|u_{\mu_{l}, \varepsilon}^{a_{l}}\right|^{q} & =\int_{B_{\delta_{0}}\left(a_{l}\right)} \frac{\varepsilon^{(q(N-2)) / 4}}{\left[\varepsilon\left|x-a_{l}\right|^{\gamma_{l}^{\prime} / \sqrt{\bar{\mu}}}+\left|x-a_{l}\right|^{\gamma_{l} / \sqrt{\bar{\mu}}}\right]^{\sqrt{\bar{\mu}} q}} \\
& \geq \int_{B_{\delta_{0}}\left(a_{l}\right)} \frac{\varepsilon^{(q(N-2)) / 4}}{\left(\left(2 \delta_{0}^{\eta / \sqrt{\bar{\mu}}}\right)^{\sqrt{\bar{\mu}} q}\right.} \\
& =C_{1}\left(N, q, \mu_{l}, \delta_{0}\right) \varepsilon^{(q(N-2)) / 4} .
\end{aligned}
$$

Combining with (4.26) and (4.27), for all $\varepsilon \in\left(0, \delta_{0}^{\left(\gamma_{l}-\gamma_{l}^{\prime}\right) / \sqrt{\bar{\mu}}}\right)$, we get

$$
\sup _{t \geq t_{0}} g(t) \leq \frac{S_{\mu_{l}}^{N / 2}}{N Q\left(a_{l}\right)^{(N-2) / 2}}+O\left(\varepsilon^{(N-2) / 2}\right)-\frac{t_{0}^{q}}{q} C_{1} \lambda \varepsilon^{(q(N-2)) / 4} .
$$

Hence, for any $\lambda>0$, we can choose small positive constant $\varepsilon_{\lambda}<\min \left\{1, \delta_{0}^{\left(\gamma_{1}-\gamma_{1}^{\prime}\right) / \sqrt{\bar{\mu}}}\right\}$ such that

$$
O\left(\varepsilon_{\lambda}{ }^{(N-2) / 2}\right)-\frac{t_{0}^{q}}{q} C_{1} \lambda \varepsilon_{\lambda}^{(q(N-2)) / 4}<0
$$

From (4.25), (4.28), and (4.29), we can deduce that, for any $\lambda>0$, there exists $\varepsilon_{\curlywedge}>0$ such that

$$
\sup _{t \geq 0} J_{\lambda}\left(t u_{\mu_{l}, \varepsilon_{\lambda}}^{a_{l}}\right)<\frac{S_{\mu_{l}}^{N / 2}}{N Q\left(a_{l}\right)^{(N-2) / 2}}
$$


From Lemma 2.4 (i), the definition of $\alpha_{\lambda}^{-}$, and (4.30), we can deduce that, for any $\lambda \in$ $\left(0, \Lambda_{0}\right)$, there exists $t_{\varepsilon_{\lambda}}>0$ such that $t_{\varepsilon_{\curlywedge}} u_{\varepsilon_{\curlywedge}} \in \mathcal{N}_{\lambda}^{-}$and

$$
\alpha_{\lambda}^{-} \leq J_{\lambda}\left(t_{\varepsilon_{\lambda}} u_{\mu_{l}, \varepsilon_{\lambda}}^{a_{l}}\right) \leq \sup _{t \geq 0} J_{\lambda}\left(t u_{\mu_{l}, \varepsilon_{\lambda}}^{a_{l}}\right)<\frac{S_{\mu_{l}}^{N / 2}}{N Q\left(a_{l}\right)^{(N-2) / 2}} .
$$

Hence Case I is verified.

Next, we investigate Case II. In this case we have that

$$
c^{*}=\frac{S_{0}^{N / 2}}{N Q_{M}^{(N-2) / 2}}=\frac{S_{0}^{N / 2}}{N Q\left(x_{0}\right)^{(N-2) / 2}} \leq \frac{S_{\mu_{l}}^{N / 2}}{N Q\left(a_{l}\right)^{(N-2) / 2}},
$$

where $x_{0}$ is the maximum point of $Q(x)$ defined as in $\left(\mathscr{t}_{2}\right)$.

If $x_{0}=a_{i}$ for some $i \in\{1,2, \ldots, k\}$, from the fact that $S_{\mu_{i}}<S_{0}$, we obtain

$$
c^{*}=\frac{S_{0}^{N / 2}}{N Q\left(a_{i}\right)^{(N-2) / 2}}>\frac{S_{\mu_{i}}^{N / 2}}{N Q\left(a_{i}\right)^{(N-2) / 2}} \geq \frac{S_{\mu_{l}}^{N / 2}}{N Q\left(a_{l}\right)^{(N-2) / 2}},
$$

which is impossible. Hence $x_{0} \neq a_{i}$ for any $i \in\{1,2, \ldots, k\}$.

For $\varepsilon>0$, let

$$
u_{0, \varepsilon}^{x_{0}}(x)=\frac{\varepsilon^{(N-2) / 4} \eta^{x_{0}}(x)}{\left(\varepsilon+\left|x-x_{0}\right|^{2}\right)^{(N-2) / 2}}
$$

where $\eta^{x_{0}}(x)$ is a cut-off function that satisfies $\eta^{x_{0}}(x) \in C_{0}^{\infty}\left(B_{2 \delta_{0}}\left(x_{0}\right)\right), \eta^{x_{0}}(x)=1$ for $\left|x-x_{0}\right|<$ $\delta_{0}, \eta^{x_{0}}(x)=0$ for $\left|x-x_{0}\right|>2 \delta_{0}, 0 \leq \eta^{x_{0}} \leq 1$ and $\left|\nabla \eta^{x_{0}}\right| \leq C$ where $0<\delta_{0}<(1 / 2) \min \left\{\mid x_{0}-\right.$ $\left.a_{1}|,| x_{0}-a_{2}|, \ldots,| x_{0}-a_{k} \mid, 2 \rho_{0}\right\}$ and $B_{2 \delta_{0}}\left(x_{0}\right) \subset \Omega$. Consider the functions defined on the interval $[0,+\infty)$ :

$$
\begin{gathered}
\bar{h}(t) \triangleq \frac{t^{2}}{2} \int_{\Omega}\left|\nabla u_{0, \varepsilon}^{x_{0}}\right|^{2}-\frac{t^{2^{*}}}{2^{*}} \int_{\Omega} Q(x)\left|u_{0, \varepsilon}^{x_{0}}\right|^{2^{*}}, \\
h(t) \triangleq J_{\lambda}\left(t u_{0, \varepsilon}^{x_{0}}\right)=\bar{h}(t)-\frac{t^{2}}{2} \sum_{i=1}^{k} \mu_{i} \int_{\Omega} \frac{\left(u_{0, \varepsilon}^{x_{0}}\right)^{2}}{\left|x-a_{i}\right|^{2}}-\lambda \frac{t^{q}}{q} \int_{\Omega}\left|u_{0, \varepsilon}^{x_{0}}\right|^{q} .
\end{gathered}
$$

By the same argument as in Case I, we can deduce that

$$
\begin{gathered}
\sup _{t \geq 0} \bar{h}(t)=\frac{S_{0}^{N / 2}}{N Q\left(x_{0}\right)^{(N-2) / 2}}+O\left(\varepsilon^{(N-2) / 2}\right), \\
\int_{\Omega}\left|u_{0, \varepsilon}^{x_{0}}\right|^{q} \geq C_{2}\left(N, q, \delta_{0}\right) \varepsilon^{q(N-2) / 4} \quad \forall \varepsilon \in\left(0, \delta_{0}^{2}\right),
\end{gathered}
$$


and, for any $\lambda>0$, there exists $0<\varepsilon_{\mathcal{\lambda}}<\min \left\{1, \delta_{0}^{2}\right\}$ such that

$$
\sup _{t \geq 0} J_{\lambda}\left(t u_{0, \varepsilon_{\lambda}}^{x_{0}}\right)<\sup _{t \geq 0}\left(\bar{h}(t)-\lambda \frac{t^{q}}{q} \int_{\Omega}\left|u_{0, \varepsilon_{\lambda}}^{x_{0}}\right|^{q}\right)<\frac{S_{0}^{N / 2}}{N Q\left(x_{0}\right)^{(N-2) / 2}}
$$

From Lemma 2.4 (i), the definition of $\alpha_{\lambda}^{-}$, and (4.37), we can deduce that, for any $\lambda \in$ $\left(0, \Lambda_{0}\right)$, there exists $t_{\varepsilon_{\curlywedge}}>0$ such that $t_{\varepsilon_{\lambda}} u_{\varepsilon_{\curlywedge}} \in \mathcal{N}_{\lambda}^{-}$and

$$
\alpha_{\lambda}^{-} \leq J_{\lambda}\left(t_{\varepsilon_{\lambda}} u_{0, \varepsilon_{\lambda}}^{x_{0}}\right) \leq \sup _{t \geq 0} J_{\lambda}\left(t u_{0, \varepsilon_{\curlywedge}}^{x_{0}}\right)<\frac{S_{0}^{N / 2}}{N Q\left(x_{0}\right)^{(N-2) / 2}} .
$$

Hence Case II is proved. From Case I and II we conclude Lemma 4.3.

Now, we establish the existence of a local minimum of $J_{\lambda}$ on $\mathcal{M}_{\lambda}^{-}$.

Theorem 4.4. Assume that condition ( $\mathscr{\ell})$ holds. If $\lambda \in\left(0,(q / 2) \Lambda_{0}\right)$, then $J_{\lambda}$ has a minimizer $U_{\lambda}$ in $\mathcal{M}_{\lambda}^{-}$, and it satisfies the following:

(i) $J_{\lambda}\left(U_{\curlywedge}\right)=\alpha_{\lambda^{\prime}}^{-}$

(ii) $U_{\curlywedge}$ is a positive solution of problem $\left(E_{\curlywedge}\right)$.

Proof. If $\lambda \in\left(0,(q / 2) \Lambda_{0}\right)$, then, by Theorem 3.1 (ii), Proposition 3.3 (ii), and Lemma 4.3, there exists a (PS) $\alpha_{\alpha_{\lambda}^{-}}$sequence $\left\{u_{n}\right\} \subset \mathcal{M}_{\lambda}^{-}$in $H$ for $J_{\lambda}$ with $\alpha_{\lambda}^{-} \in\left(0, c^{*}\right)$. From Lemma 4.2 , there exist a subsequence still denoted by $\left\{u_{n}\right\}$ and a nonzero solution $U_{\lambda} \in H$ of problem $\left(E_{\lambda}\right)$ such that $u_{n} \rightarrow U_{\lambda}$ weakly in $H$. Now we prove that $u_{n} \rightarrow U_{\lambda}$ strongly in $H$ and $J_{\lambda}\left(U_{\lambda}\right)=\alpha_{\lambda}^{-}$. By (3.18), if $u \in \mathcal{M}_{\lambda}$, then

$$
J_{\lambda}(u)=\frac{1}{N}\|u\|^{2}-\lambda \frac{2^{*}-q}{2^{*} q} \int_{\Omega}|u|^{q} .
$$

First, we prove that $U_{\lambda} \in \mathcal{M}_{\lambda}^{-}$. On the contrary, if $U_{\curlywedge} \in \mathcal{M}_{\lambda}^{+}$, then by, the definition of

$$
\mathcal{M}_{\lambda}^{-}=\left\{u \in \mathcal{M}_{\lambda}: \varphi_{u}^{\prime \prime}(1)<0\right\}
$$

and Lemma 2.3, we have $\left\|U_{\lambda}\right\|^{2}<\liminf _{n \rightarrow \infty}\left\|u_{n}\right\|^{2}$. By Lemma 2.4 (i), there exists a unique $t_{\lambda}^{-}$such that $t_{\lambda}^{-} U_{\lambda} \in \mathcal{M}_{\lambda}^{-}$. Since $u_{n} \in \mathcal{M}_{\lambda}^{-}$, by (3.12) and (4.39), we have $J_{\lambda}\left(u_{n}\right) \geq J_{\lambda}\left(t u_{n}\right)$ for all $t \geq 0$ and

$$
\alpha_{\lambda}^{-} \leq J_{\lambda}\left(t_{\lambda}^{-} U_{\lambda}\right)<\liminf _{n \rightarrow \infty} J_{\lambda}\left(t_{\lambda}^{-} u_{n}\right) \leq \liminf _{n \rightarrow \infty} J_{\lambda}\left(u_{n}\right)=\alpha_{\lambda^{\prime}}^{-}
$$

and this is a contradiction. 
In order to prove that $J_{\lambda}\left(U_{\lambda}\right)=\alpha_{\lambda}^{-}$, it suffices to recall that $u_{n}, U_{\lambda} \in \mathcal{M}_{\lambda}^{-}$for all $n$, by (4.39) and applying Fatou's lemma to get

$$
\begin{aligned}
\alpha_{\lambda}^{-} & \leq J_{\lambda}\left(U_{\lambda}\right)=\frac{1}{N}\left\|U_{\lambda}\right\|^{2}-\lambda \frac{2^{*}-q}{2^{*} q} \int_{\Omega}\left|U_{\lambda}\right|^{q} \\
& \leq \liminf _{n \rightarrow \infty}\left(\frac{1}{N}\left\|u_{n}\right\|^{2}-\lambda \frac{2^{*}-q}{2^{*} q} \int_{\Omega}\left|u_{n}\right|^{q}\right) \\
& \leq \liminf _{n \rightarrow \infty} J_{\lambda}\left(u_{n}\right)=\alpha_{\lambda}^{-} .
\end{aligned}
$$

This implies that $J_{\lambda}\left(U_{\lambda}\right)=\alpha_{\lambda}^{-}$and $\lim _{n \rightarrow \infty}\left\|u_{n}\right\|^{2}=\left\|U_{\lambda}\right\|^{2}$. Let $v_{n}=u_{n}-U_{\lambda}$; then Brézis-Lieb's lemma [18] implies that

$$
\left\|v_{n}\right\|^{2}=\left\|u_{n}\right\|^{2}-\left\|U_{\lambda}\right\|^{2}+o_{n}(1)
$$

Therefore, $u_{n} \rightarrow U_{\curlywedge}$ strongly in $H$.

Since $J_{\lambda}\left(U_{\lambda}\right)=J_{\lambda}\left(\left|U_{\lambda}\right|\right)=\alpha_{\lambda}^{-}$and $\left|U_{\lambda}\right| \in \mathcal{M}_{\lambda}^{-}$, by Lemma 2.2, we may assume that $U_{\lambda}$ is a nonzero nonnegative solution of problem $\left(E_{\curlywedge}\right)$. Finally, by the Harnack inequality [19], we deduce that $U_{\lambda}>0$ in $\Omega$.

Now, we complete the proof of Theorem 1.1. By Theorems 3.4 and 4.4, we obtain that problem $\left(E_{\lambda}\right)$ has two positive solutions $u_{\lambda}$ and $U_{\lambda}$ such that $u_{\lambda} \in \mathcal{M}_{\lambda}^{+}, U_{\lambda} \in \mathcal{M}_{\lambda}^{-}$. Since $\mathcal{M}_{\lambda}^{+} \cap \mathcal{M}_{\lambda}^{-}=\emptyset$, this implies that $u_{\lambda}$ and $U_{\lambda}$ are distinct. This completes the proof of Theorem 1.1.

\section{References}

[1] F. Catrina and Z.-Q. Wang, "On the Caffarelli-Kohn-Nirenberg inequalities: sharp constants, existence (and nonexistence), and symmetry of extremal functions," Communications on Pure and Applied Mathematics, vol. 54, no. 2, pp. 229-258, 2001.

[2] K. S. Chou and C. W. Chu, "On the best constant for a weighted Sobolev-Hardy inequality," Journal of the London Mathematical Society, vol. 48, no. 1, pp. 137-151, 1993.

[3] A. Ambrosetti, J. Garcia-Azorero, and I. Peral, "Multiplicity results for some nonlinear elliptic equations," Journal of Functional Analysis, vol. 137, no. 1, pp. 219-242, 1996.

[4] M. Bouchekif and A. Matallah, "Multiple positive solutions for elliptic equations involving a concave term and critical Sobolev-Hardy exponent," Applied Mathematics Letters, vol. 22, no. 2, pp. 268-275, 2009.

[5] J. Chen, "Multiple positive solutions for a class of nonlinear elliptic equations," Journal of Mathematical Analysis and Applications, vol. 295, no. 2, pp. 341-354, 2004.

[6] T.-S. Hsu and H.-L. Lin, "Multiple positive solutions for singular elliptic equations with concaveconvex nonlinearities and sign-changing weights," Boundary Value Problems, vol. 2009, Article ID 584203, 17 pages, 2009.

[7] T.-S. Hsu and H.-L. Lin, "Multiple positive solutions for singular elliptic equations with weighted Hardy terms and critical Sobolev-Hardy exponents," Proceedings of the Royal Society of Edinburgh. Section A, vol. 140, no. 3, pp. 617-633, 2010.

[8] D. Cao and P. Han, "Solutions to critical elliptic equations with multi-singular inverse square potentials," Journal of Differential Equations, vol. 224, no. 2, pp. 332-372, 2006.

[9] V. Felli and S. Terracini, "Elliptic equations with multi-singular inverse-square potentials and critical nonlinearity," Communications in Partial Differential Equations, vol. 31, no. 1-3, pp. 469-495, 2006.

[10] T. S. Hsu, "Multiple positive solutions for semilinear elliptic equations involving multi-singular inverse square potentials and concave-convex nonlinearities," Nonlinear Analysis: Theory, Methods $\mathcal{E}$ Applications, In press. 
[11] A. Ferrero and F. Gazzola, "Existence of solutions for singular critical growth semilinear elliptic equations," Journal of Differential Equations, vol. 177, no. 2, pp. 494-522, 2001.

[12] P. Drábek and S. I. Pohozaev, "Positive solutions for the $p$-Laplacian: application of the fibering method," Proceedings of the Royal Society of Edinburgh. Section A, vol. 127, no. 4, pp. 703-726, 1997.

[13] K. J. Brown and Y. Zhang, "The Nehari manifold for a semilinear elliptic equation with a signchanging weight function," Journal of Differential Equations, vol. 193, no. 2, pp. 481-499, 2003.

[14] P. A. Binding, P. Drábek, and Y. X. Huang, "On Neumann boundary value problems for some quasilinear elliptic equations," Electronic Journal of Differential Equations, p. No. 05, approx. 11 pp. (electronic), 1997.

[15] T.-F. Wu, "Multiple positive solutions for a class of concave-convex elliptic problems in $\mathbb{R}^{N}$ involving sign-changing weight," Journal of Functional Analysis, vol. 258, no. 1, pp. 99-131, 2010.

[16] I. Ekeland, "On the variational principle," Journal of Mathematical Analysis and Applications, vol. 47, pp. 324-353, 1974.

[17] T.-F. Wu, “On semilinear elliptic equations involving concave-convex nonlinearities and signchanging weight function," Journal of Mathematical Analysis and Applications, vol. 318, no. 1, pp. 253$270,2006$.

[18] H. Brézis and E. Lieb, "A relation between pointwise convergence of functions and convergence of functionals," Proceedings of the American Mathematical Society, vol. 88, no. 3, pp. 486-490, 1983.

[19] N. S. Trudinger, "On Harnack type inequalities and their application to quasilinear elliptic equations," Communications on Pure and Applied Mathematics, vol. 20, pp. 721-747, 1967.

[20] P.-L. Lions, "The concentration-compactness principle in the calculus of variations. The limit case. I," Revista Matemática Iberoamericana, vol. 1, no. 1, pp. 145-201, 1985.

[21] P.-L. Lions, "The concentration-compactness principle in the calculus of variations. The limit case. II," Revista Matemática Iberoamericana, vol. 1, no. 2, pp. 45-121, 1985.

[22] J. Chen, "Existence of solutions for a nonlinear PDE with an inverse square potential," Journal of Differential Equations, vol. 195, no. 2, pp. 497-519, 2003. 


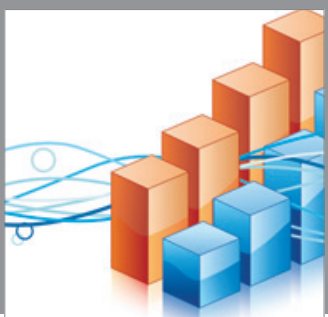

Advances in

Operations Research

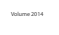

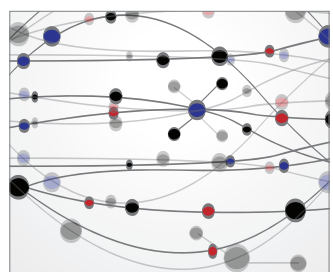

\section{The Scientific} World Journal
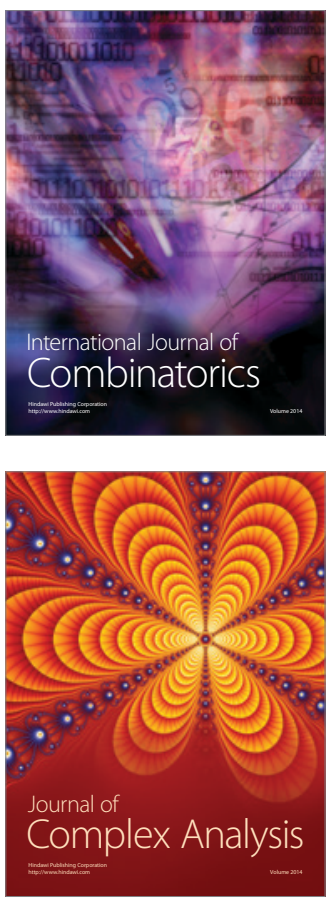

International Journal of

Mathematics and

Mathematical

Sciences
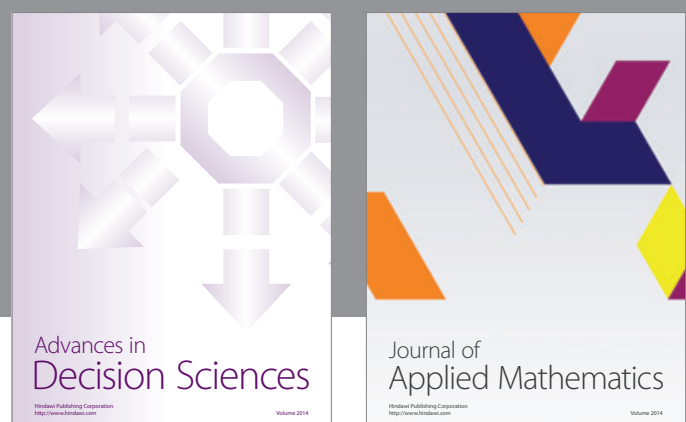

Journal of

Applied Mathematics
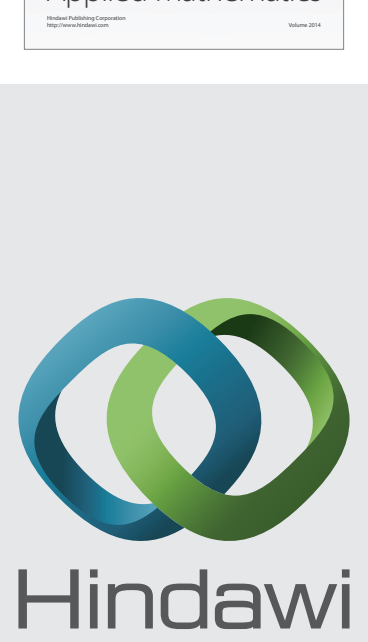

Submit your manuscripts at http://www.hindawi.com
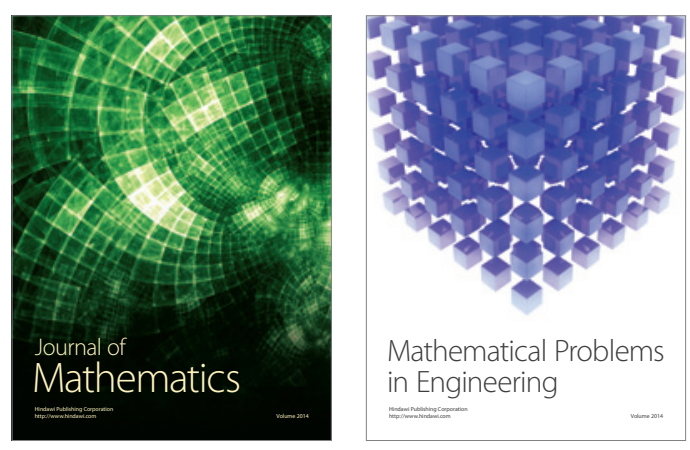

Mathematical Problems in Engineering
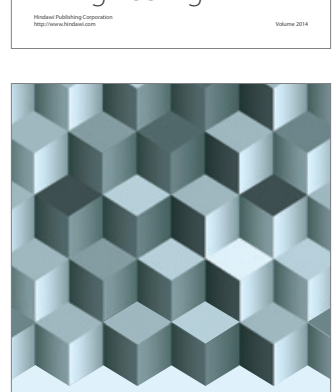

Journal of

Function Spaces
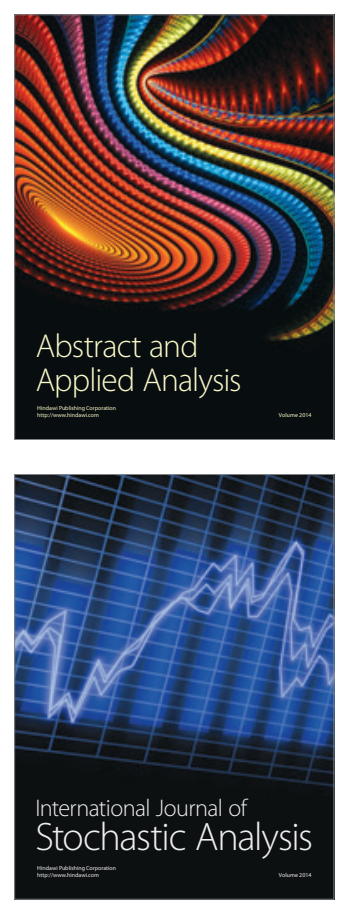

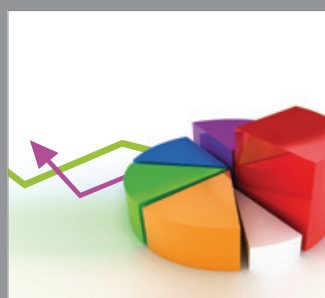

ournal of

Probability and Statistics

Promensencen
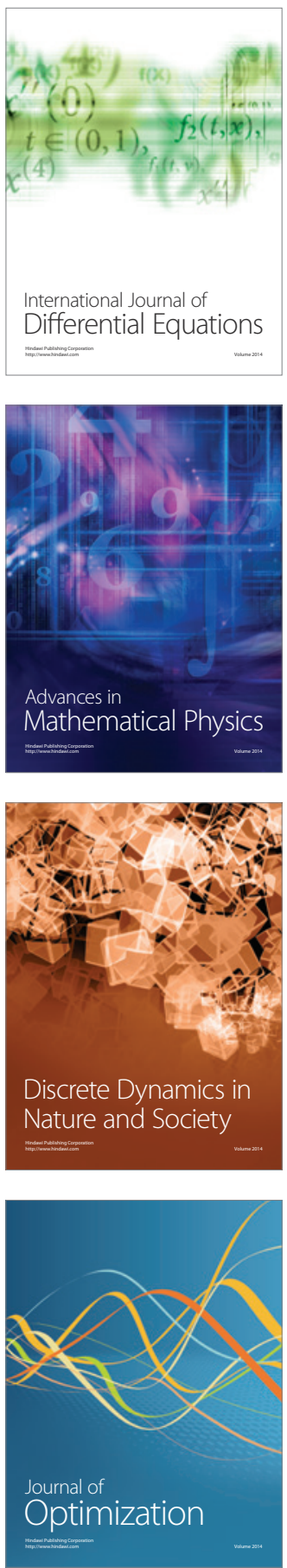